\title{
Cortisol profiles: A test for adaptive calibration of the stress response system in maltreated and nonmaltreated youth
}

\author{
MELISSA K. PECKINS ${ }^{a}$, ELIZABETH J. SUSMAN ${ }^{a, b}$, SONYA NEGRIFF ${ }^{b}$, JENNIE NOLL ${ }^{a}$, and \\ PENELOPE K. TRICKETT' \\ aPennsylvania State University \\ bUniversity of Southern California
}

\begin{abstract}
Throughout the life span, exposure to chronic stress such as child maltreatment is thought to contribute to future dysfunction of the stress response system (SRS) through the process of adaptive calibration. Dysfunction of the SRS is associated with numerous health and behavior problems, so it is important to understand under what conditions and what time frame adaptive calibration occurs. The present study tested for adaptive calibration of the SRS in a sample of maltreated $(n=303)$ and nonmaltreated $(n=151)$ youth during the important developmental period of adolescence. Data were used from Waves 2, 3, and 4 of a larger study of the consequences of maltreatment on health and well-being. At each time point, participants underwent the Trier Social Stress Test for Children and provided a baseline and four poststressor saliva samples to measure cortisol reactivity. Adaptive calibration was tested by performing a latent profile analysis using the five samples of salivary cortisol provided at each time point, and testing whether maltreatment status predicted the likelihood of profile membership at Time 2, Time 3, and Time 4. Three cortisol profiles emerged from the data at each time point (blunted, moderate, and elevated), and results indicated that maltreated youth were more likely than nonmaltreated youth to present with the blunted cortisol profile compared to the moderate and elevated profiles at Time 2 and Time 3, even after controlling for recent exposure to violence and trauma. At Time 4, there was no longer a difference in profile membership between maltreated and nonmaltreated youth, suggesting adaptive calibration may be a lengthy process requiring a period of years to become evident. Overall, the findings provide support for adaptive calibration and offer insight into the conditions under which adaptive calibration occurs.
\end{abstract}

Starting in utero, the progression through development is marked with a myriad of challenges or stressors that shape an individual's behavioral and physiological relationship with the social and physical environment. This relationship is ongoing; individuals are constantly interacting with their environment by absorbing and processing new information in order to formulate appropriate physiological and behavioral responses to stress (McEwen, 2007). Over time, the process of allostasis, or adapting to stress (Sterling \& Eyer, 1988) via repeated activation of the stress response system (SRS), is thought to deleteriously influence

Address correspondence and reprint requests to: Melissa K. Peckins, Bennett Pierce Prevention Research Center, Pennsylvania State University, 314 Biobehavioral Health Building, University Park, PA 16802; mkp138@psu.edu. 
individuals' physiological responses to future stressors leading to dysfunction (i.e., hypo- or hyperactivity) of the SRS. Both hypo- and hyperactivity of the SRS have alarming implications for health. Dysfunction of the SRS is associated with health problems, including depression and obesity (for review, see Tsigos \& Chrousos, 2002). As a result of repeated cycles of allostasis and dysfunction of the SRS, allostatic load (AL) accumulates, manifesting as pathological outcomes including cardiovascular disease and autoimmune disease (McEwen, 1998). One underlying tenet of AL is that the consequences of chronic activation on SRS function are cumulative (McEwen, 2000a, 2000b); however, up until recently (McEwen, Gray, \& Nasca, 2015), there has been no discussion on whether the damaging effects of repeated cycles of allostasis (e.g., chronic stress) on functioning of the SRS are reversible as indicated by adaptation of the SRS. In order to better understand the potential for reversibility of SRS dysfunction as a result of chronic stress, the adaptive calibration model (ACM; Del Giudice, Ellis, \& Shirtcliff, 2011) is considered.

The ACM is one theory that explains individual differences in functioning of the SRS and suggests that adaptive calibration of the SRS takes place to meet the demands of a particular environment while considering the cost to fitness (for review, see Del Giudice et al., 2011). The ACM postulates that through adaptive calibration of the SRS, individuals will display one of four SRS patterns: sensitive (moderate basal activity, high reactivity), buffered (moderate basal activity, high reactivity), vigilant (moderate to high basal activity, high reactivity), or unemotional (low basal activity, low reactivity). The four SRS patterns describe individuals' basal functioning and reactivity of the SRS along a spectrum ranging from low to high activity (Del Giudice et al., 2011), and whichever pattern an individual displays is thought to be adaptive to the individual's current social and physical environment. Individuals may display one pattern of SRS activity at one point in time, but as their environmental conditions change, adaptive calibration is expected to take place to meet current environmental demands and individuals may shift to another pattern of SRS activity.

There is growing evidence supporting the underlying tenets of the ACM in adults. For example, from childhood to young adulthood, females with a history of child sexual abuse transitioned from elevated to attenuated levels of morning cortisol across development, whereas females who were not sexually abused maintained a similar pattern of normative morning levels across development (Trickett, Noll, Susman, Shenk, \& Putnam, 2010). There is some evidence for adaptive calibration in youth; although, to date, the vast majority of studies test for dysfunction of the cortisol awakening response and diurnal change in cortisol at one point in time. For example, of youth experiencing high levels of internalizing problems, children with a history of early physical and sexual abuse presented with an attenuated decline in cortisol levels across the day compared to maltreated children without a history of early physical and sexual abuse and nonmaltreated comparison youth (Cicchetti, Rogosch, Gunnar, \& Toth, 2010). There is limited support for an ACM of cortisol reactivity in youth; however, maltreated adolescents exhibited attenuated cortisol reactivity compared to nonmaltreated adolescents at one point in time (Trickett, Gordis, Peckins, \& Susman, 2014), and community violence exposure was associated with attenuated cortisol reactivity across 1 year in male children (Peckins, Dockray, Eckenrode, Heaton, \& Susman, 2012). Attenuated levels can be attributed to chronic exposure to stressors acting at the receptor or hypothalamic-pituitary-adrenal (HPA) axis levels. From an ACM perspective, the finding of 
HPA axis dysfunction is considered to be adaptive in situations where a child is exposed to chronic stress such as maltreatment or violence. However, because dysfunction of the HPA axis is linked with a variety of health conditions (Tsigos \& Chrousos, 2002) and is consequently associated with poorer overall daily functioning, understanding the mechanisms whereby adaptive calibration takes place becomes increasingly important for the well-being of youth as they transition into young adulthood.

Despite the evidence supporting the underlying tenets of the ACM for the SRS in youth and adults, it is unclear under what conditions and what time frame adaptive calibration of the SRS takes place. For example, in the study of sexually abused females presented previously, the shift from hyper- to hypocortisolism took place over 16 years. Unfortunately, most of the evidence supporting adaptive calibration in youth has used only one time point, which limits the ability to examine shifting patterns of HPA activity. Furthermore, childhood and adolescence are especially important periods of development for examining the neurobiology of the SRS, because during this time, the various components of the HPA axis are rapidly developing (Lupien, McEwen, Gunnar, \& Heim, 2009; Romeo \& McEwen, 2006) and environmental challenges that take place during this period may have a more pronounced effect on the crystallization of HPA axis function. To date, there are no studies that capture the transitional period of adolescence when vulnerabilities may emerge or be enhanced.

\section{Hypotheses}

Previous studies have provided limited evidence for adaptive calibration across adolescence. In addition, none have tested different types of maltreatment in relation to the hypothesized SRS patterns described by the ACM. Therefore, the present study used three waves of data from middle to late adolescence to test for adaptive calibration of the SRS in maltreated and nonmaltreated youth via the production of cortisol in response to a stressor (because the biomarker cortisol can be easily measured in saliva and reliably elevated in response to a laboratory stressor). It was hypothesized that consistent with the ACM, four distinct patterns of stress reactivity would emerge from the data: sensitive, buffered, vigilant, and unemotional. Based on previous research (e.g., Trickett et al., 2014), it was hypothesized that maltreated youth would be more likely than nonmaltreated youth to present with the unemotional (i.e., blunted) pattern of stress reactivity at each occasion of measurement. A blunted pattern was expected to be present in maltreated youth regardless of maltreatment type.

\section{Methods}

\section{Sample characteristics}

The present study used data from the second, third, and fourth time points of a longitudinal study of the effects of maltreatment on the health and well-being of adolescents. At Time 1 (T1), the sample was composed of 454 adolescents ( $n=212$ females) ages 9-12 years $(M=$ 10.95 years). Time 2 (T2), Time 3 (T3), and Time 4 (T4) occurred approximately $1,2.5$, and 4.5 years after baseline, respectively. The mean age of youth at T2, T3, and T4 was 12.11, 13.69 , and 18.23 years, respectively. The diverse sample of youth identified as being Black 
or African American $(n=171)$, Hispanic $(n=177)$, White/non-Hispanic $(n=50)$, and biracial $(n=56)$.

\section{Sample recruitment and retention}

Maltreated youth ( $n=303)$ were recruited from active cases in the Children and Family Services (CFS) of a large West Coast city. The inclusion criteria were (a) a new substantiated referral to CFS in the preceding month for any type of maltreatment (e.g., neglect, physical abuse, or sexual abuse); (b) child age of 9 to 12 years; (c) child identified as Hispanic, African American/Black, or Caucasian (non-Hispanic); and (d) child residing in 1 of 10 zip codes in a designated county at the time of referral to CFS. With approval of CFS and the institutional review board of the affiliated university, potential participants were contacted via postcard and asked to indicate their willingness to participate. Contact via e-mail was followed by a phone call. Of all parents/guardians contacted, 77\% provided assent (child) and consent (parent/guardian) to participate in the study. A certificate of confidentiality was obtained from the federal government to protect each participant's confidentiality; however, disclosures of maltreatment that were not already reported to a CFS agency were not protected under this agreement.

The comparison group $(n=151)$ was recruited using names from school lists of children aged 9-12 years residing in the same 10 zip codes as the maltreated sample. Comparison caregivers were contacted the same way as the maltreated group. Comparison families were cross-checked through the CFS database to ensure they had no previous or ongoing experience with child welfare agencies. Approximately $50 \%$ of the comparison families contacted agreed to participate.

Participants were assessed at four occasions of measurement, and retention strategies (e.g., contact through back-up contact information and social media) were effective because the retention rate between $\mathrm{T} 1$ and T2 was $86.1 \%(n=391)$, between $\mathrm{T} 1$ and T3 was $70.9 \%(n=$ $322)$, and between $\mathrm{T} 1$ and $\mathrm{T} 4$ was $77.5 \%(n=352)$.

Procedure

All four assessments were conducted at an urban research university and all procedures were approved by the institutional review board of the affiliated university. After assent and consent by the participant and a parent/guardian, adolescents completed a 4-hr protocol, which included collection of saliva as well as cognitive, behavioral, and health assessments. Only a subset of the measures were used in the following analyses. Child and parent/ guardian participants were compensated according to the National Institutes of Health Normal Volunteer Program.

Measures

Cortisol reactivity-Cortisol reactivity was measured in response to the Trier Social Stress Test for Children (TSST-C; Kirschbaum, Pirke, \& Hellhammer, 1993). The TSST-C is a social and cognitive stressor consisting of two tasks: a story completion task and a mental, serial subtraction ask, each lasting 4 min. Participants were told prior to the story completion task that their performance would be compared to the performances of their peers. 
Participants were read a story stem, given $5 \mathrm{~min}$ to prepare an ending, and then spent 4 min presenting the story to a panel of two judges and the interviewer. If the participant paused during the story completion task, participants were prompted up to five times to "keep going" (each prompt was unique). At the end of $4 \mathrm{~min}$, participants were then asked to complete an age-graded mental serial subtraction task for $4 \mathrm{~min}$. If participants reported an incorrect number, they were stopped and told to start from their last correct answer. The TSST-C was performed at all four waves of assessment, but for the present study, only the data from $\mathrm{T} 2, \mathrm{~T} 3$, and $\mathrm{T} 4$ were used.

At each wave of data collection, participants provided six saliva samples by spitting into a 5$\mathrm{ml}$ tube to measure cortisol reactivity in response to the TSST-C. Two baseline samples of saliva were collected, the first immediately upon arrival after consent/assent forms had been signed (at T -45 min TSST-C) and the second immediately following a 5-min relaxation period (at T-10 min TSST-C) during which the child watched a relaxing computer image and listened to relaxing music. Four poststressor saliva samples were collected; one sample immediately following the stressor (+0 TSST-C) and three samples at 10-min increments (+10 min, $+20 \mathrm{~min}$, and +30 min TSST-C). Immediately following each saliva collection, samples were frozen and sent to Salimetrics Inc. (State College, PA), where they were stored at $-80{ }^{\circ} \mathrm{C}$ until they were assayed for cortisol. Samples were assayed in duplicate, and the mean for each sample was used in analyses. Cortisol samples were log transformed to adjust for positive skew. Log transformed cortisol samples were used in all subsequent analyses. Only saliva samples 2 through 6 were used in the analysis to account for the effect of the lab, novelty of the experience on Sample 1.

Maltreatment status and type-Maltreatment status was confirmed upon admittance to the study by the child having a recent Los Angeles Department of Child and Family Services referral. Maltreatment type was determined by a careful review of Los Angeles Department of Child and Family Services records by a PhD social work student who recorded whether the child experienced at least one incident of sexual abuse, physical abuse, emotional abuse, or neglect according to the Maltreatment Classification System (Barnett, Manly, \& Cicchetti, 1993; Mennen, Kim, Sang, \& Trickett, 2010). Children were then categorized according to their history of sexual abuse and physical abuse given that sexual and physical abuse are more likely to qualify as traumatic experiences (Leeb, Paulozzi, Melanson, Simon, \& Arias, 2008) by eliciting feelings of fear and/or helplessness and by causing actual or threat of harm. Children who experienced sexual and/or physical abuse, regardless of their emotional abuse and neglect experiences, were classified into one category: sexually/physically abused. All children who experienced either emotional abuse and/or neglect and did not experience sexual and/or physical abuse were classified into a second category: emotionally abused/ neglected. This resulted in two mutually exclusive maltreatment categories, which were also used by Trickett et al. (2014).

Covariates-The Community Violence Index (adapted by Margolin \& Gordis, 2000; Richters \& Saltzman, 1990) is a self-report questionnaire used to assess recent exposure to violence (i.e., past 12 months) in this sample of youth. The Community Violence Index assessed the frequency at which the adolescent personally witnessed 19 different violent 
events (e.g., seeing someone stabbed with a knife) in the past year in his or her community, school, or place of work. A sum of all recent exposures occurring in the past year at T2, T3, and $\mathrm{T} 4$ were used in the analyses.

The Comprehensive Trauma Interview (Horowitz, 1998; Noll, Horowitz, Bonanno, Trickett, \& Putnam, 2003) was used to assess recent exposure to traumatic experiences at T3 and T4. The Comprehensive Trauma Interview assesses 19 different potentially traumatic experiences, including maltreatment experiences, and includes an upset rating on a scale of 1 to 5, 1 being not upset at all, which was used to establish whether or not the event met the criteria to be considered traumatic (i.e., eliciting feelings of fear or helplessness). If the child assigned a upset rating of 2 to 5 to any event that occurred in the past year, it was considered to be a traumatic experience. Adolescents provided the age each experience occurred; if the trauma was within 1 year of their age at the time of assessment, it was included in the sum score for the scale.

The time-varying covariate age was determined by calculating the number of days between the child's date of birth and the interview date at each wave of measurement, then transforming the child's age from days to age in years (rounded to the nearest hundredth decimal place). Age was considered to be a proxy for pubertal development because the two are highly correlated (Nottelmann et al., 1987) and most children age out of pubertal staging (i.e., Tanner Stage 5) by age 17 (Marceau, Ram, Houts, Grimm, \& Susman, 2011; Slyper, 2006). Race was reported by the child's parent or guardian at T1 as being Black/African American, White (non-Hispanic), or biracial. In the United States, the largest percentage of victimized youth identified as being White (43.9\%; US Department of Health and Human Services, Administration for Children and Families, Administration on Children, Youth and Families, Children's Bureau, 2013); therefore, in subsequent analyses, child's race was regrouped as being either White (non-Hispanic) or an ethnic minority (Black/African American, Hispanic, or biracial). Time of day the participant visited the lab at each wave of assessment was also controlled for in the models to account for the normative decline in cortisol that takes place across the day in the majority of individuals (Stone et al., 2001), which may influence baseline and reactive cortisol concentrations. Children were grouped according to when they were scheduled to visit the lab: between 12 a.m. and 4 p.m. or between 4 p.m. and 8 p.m. The majority of children at all occasions of measurement were scheduled to visit the lab before 4 p.m., and on average, children arriving prior to 4 p.m. had significantly greater cortisol concentrations than children arriving at or after 4 p.m.

\section{Analytic plan}

Only data from T2, T3, and T4 were used in the analyses in order to be able to control for the effects of recent adversity (e.g., recent exposure to violence) on HPA axis activity. Recent exposure to violence and trauma were not assessed at $\mathrm{T} 1$ due to ethical and methodological considerations (e.g., age appropriateness of the questionnaire). Descriptive analyses were performed for all variables. Independent $t$ tests were performed to test for mean differences between maltreated and nonmaltreated youth in recent exposure to violence, recent exposure to trauma, and cortisol concentrations. Hypotheses were tested with a latent profile analysis (LPA) in Mplus (Muthén \& Muthén, 2007; 1998-2011). 
Missing data were handled with maximum likelihood estimation with robust standard errors. The number of cortisol reactivity patterns in the data at T2, T3, and T4 were determined by performing LPA with two-, three-, and four-profile solutions. This range of profiles was selected based on the ACM's theoretical formulation that four patterns of HPA activity exist (i.e., sensitive, buffered, vigilant, and unemotional). After the best profile solution was selected based on indices of fit, the theoretical rationale, and patterns that emerged from the data, maltreatment status (maltreated or comparison) and maltreatment type (sexual/physical abuse, emotional abuse/neglect, or comparison) were added to the LPA to test whether membership to a specific cortisol reactivity profile was predicted by maltreatment status and type. The child's recent exposure to violence, recent exposure to traumatic experiences, sex, age, race, and time of day at which the child visited the lab were also included in the analysis as covariates.

\section{Results \\ Descriptive analyses}

Frequencies and percentages for all categorical variables are provided in Table 1. Descriptive analyses were performed and are presented in Table 2. Maltreated adolescents presented with lower levels of cortisol than their nonmaltreated peers across all samples at T2 and T3 $(p<.05)$.

\section{Hypothesis tests}

LPA-LPA was performed without covariates to determine the number of cortisol profiles present in the data at T2, T3, and T4. Although inconsistent with the ACM, the three-profile solution was judged to be the best solution at all three time points based on theory, indices of fit, and classification quality (Table 3). The ACM suggests that four patterns of HPA axis activity exist, yet there was not a fourth distinct profile present in the data. However, at each wave of measurement, three similar profiles emerged from the data: a blunted cortisol profile, a moderate cortisol profile, and an elevated cortisol profile (Figure 1). Over 50\% of the youth belonged to the moderate cortisol profile at all waves of measurement, and from $\mathrm{T} 3$ to T4 the percentage of youth belonging to the elevated cortisol profile noticeably increased whereas the percentage of youth belonging to the blunted cortisol profile markedly decreased (Table 4). The three cortisol profiles were relatively stable across samples and map most closely to the unemotional (low basal activity, low reactivity), buffered (moderate basal activity, moderate reactivity), and vigilant (high basal activity, high reactivity) patterns described by the ACM (Del Giudice et al., 2011). The sensitive pattern was not found in the present data. On average, for youth presenting with moderate or elevated cortisol profiles, the minimum cortisol concentration was at baseline (Sample 2) followed by an increase in cortisol output immediately following the stressor (Sample 3), during which time cortisol output continued to increase until approximately 10 to 20 min poststressor (Samples 4 and 5), at which time cortisol output peaked and began to return to prestressor levels (Sample 6). In contrast, individuals exhibiting the blunted cortisol profile presented with low cortisol levels across all samples with no clear baseline, reactivity, or recovery period, which is indicative of SRS dysfunction. 
LPA with predictors-Predictors were added to the three-profile solution to test for adaptive calibration at T2, T3, and T4. It was expected that youth with a history of maltreatment compared to nonmaltreated youth would be more likely to exhibit the blunted cortisol profile in comparison to the moderate or elevated cortisol profiles. The blunted cortisol profile was selected to be the referent profile in the LPA because the moderate and elevated profiles are what one would expect to see in youth in response to a stressor.

The results from the LPA with maltreatment status (Table 5) and maltreatment type (Table 6) support the ACM. Maltreated youth were more likely than nonmaltreated youth to belong to the blunted cortisol profile than the moderate or elevated cortisol profiles at T2 and T3 after controlling for recent exposure to violence and trauma. The findings were also consistent for maltreatment type. Youth classified as sexually/physically abused were more likely than nonmaltreated youth to belong to the blunted cortisol profile than the moderate (T2 and T3) and elevated (T2 and T3) cortisol profiles. Similarly, emotionally abused/neglected youth without a history of sexual or physical abuse were more likely than nonmaltreated youth to belong to the blunted cortisol profile than the moderate (T2 and T3) and elevated (T2) cortisol profiles.

\section{Discussion}

Throughout the course of development from infancy into adulthood and beyond, individuals interact with their social and physical environment by absorbing and interpreting relevant information in order to navigate psychological and physical demands. The SRS, primarily mediated through functioning of the HPA axis, is adept at processing environmental stimuli, yet previous research has shown repeated activation of the SRS, as is the case with exposure to acute and chronic stressors such as maltreatment, to be associated with future dysfunction (i.e., hypo- or hyperactivity) of the SRS (McEwen, 2007) and declines in mental and physical health (for review, see Tsigos \& Chrousos, 2002). The ACM (Del Giudice et al., 2011) explains dysfunction of the SRS as the body's way of adapting and calibrating according to the social and physical demands of the individual's environment, yet it is unclear under what conditions and what time span adaptive calibration takes place, particularly in children and adolescents. The present study tested whether the ACM was applicable to a sample of maltreated and nonmaltreated youth across middle to late adolescence. Adaptive calibration of the SRS was tested by determining whether four distinct cortisol profiles (i.e., sensitive, buffered, vigilant, and unemotional) proposed by Del Giudice et al. (2011) were present in the data and whether maltreated youth were more likely than nonmaltreated youth to present with the unemotional (blunted) cortisol profile.

An important distinction between the ACM and the findings from the present analysis is that the ACM posits that four patterns of HPA axis activity exist, yet only three profiles emerged from the current data. While inconsistent with the ACM, the three cortisol profiles (i.e., blunted, moderate, and elevated) are plausible from a theoretical standpoint based on existing literature. More specifically, the three distinct profiles that were present at all three waves of data collection suggest that, on average, an adolescent's baseline cortisol is blunted, moderate, or elevated in relation to his or her peers and will follow either the typical 
cortisol response to a stressor (i.e., increase followed by a gradual decrease) or an overall attenuated cortisol response.

A history of maltreatment was associated with an increased likelihood of exhibiting the blunted cortisol profile in comparison to the moderate and elevated profiles, and this pattern was consistent for both sexually/physically abused youth and emotionally abused/neglected youth, but only at $\mathrm{T} 2$ and $\mathrm{T} 3$. In addition to the theory of AL, which suggests chronic stress contributes to future dysfunction of the SRS (McEwen, 1998), this finding is also consistent with the attenuation hypothesis (Susman, 2006), postulating that adversity experienced during youth primes the child for a future blunted or attenuated stress response. The finding that maltreatment is associated with the blunted cortisol profile is also consistent with the unemotional response as described by the ACM that presents in individuals who are habitually exposed to adversity (Del Giudice et al., 2011). The present findings suggest the experience of maltreatment may lead to downregulation of HPA axis function, which is considered adaptive because downregulation limits the body's exposure to the glucocorticoid cortisol in times of chronic stress. The findings for maltreatment status and type were not present at T4, suggesting that if adaptive calibration of the SRS occurred by T4, it may present in this sample as maltreated youth transitioning from the blunted cortisol profile to the moderate or elevated cortisol profiles at some point between T3 and T4. This is supported by the data because the percentage of youth belonging to the blunted profile markedly decreased from $39 \%$ at T3 to $19 \%$ at T4.

A history of maltreatment but not recent (i.e., past year) exposure to violence and traumatic experiences differentiated between profile membership at $\mathrm{T} 2$ and $\mathrm{T} 3$. There are several important implications from this finding in regard to the time frame and conditions under which adaptive calibration occurs. It may be that a model of cumulative risk (i.e., lifetime exposure) is more appropriate than a model of recent exposure in differentiating between cortisol profiles. It may also be that adaptive calibration is a lengthy process requiring a period of years for effects of cortisol output in response to a laboratory stressor to be observable, as was the case in the study of females with a history of sexual abuse (Trickett et al., 2010). The notion that adaptive calibration is a lengthy process is supported by the finding that likelihood of cortisol profile membership no longer differed between maltreated and nonmaltreated youth by $\mathrm{T} 4$.

The results presented in this report are novel in that they describe how the experience of maltreatment during childhood is associated with functioning of the SRS over time, yet there are several limitations worthy of discussion. LPA is an analytic technique that is both highly exploratory and highly subjective in terms of determining the number of latent profiles that are present in the data, in the assignment of meaningful labels to the profiles that accurately describe profile members, and in the language used to discuss the profiles and profile membership. The exploratory and subjective nature of the analysis is addressed in this study primarily through three means: by using a theory-driven approach to supplement statistical indicators of fit in the selection of a solution, by labeling each of the profiles that emerged from the data in relation to the remaining two profiles, and by describing the three profiles as being representative of the study sample rather than suggesting these profiles are representative of all children and adolescents. 
Another consideration is whether the present analysis is actually a test of the ACM. Longitudinal data on functioning of the SRS, particularly cortisol reactivity in children and adolescents, is sparse, and consequently, it is not known on what time scale adaptive calibration takes place, if at all, in children and adolescents who are maltreated. Maltreatment status and type were associated with having a blunted cortisol reactivity pattern after controlling for recent exposure to violence and trauma, sex, race, age, and time of visit, suggesting that there is at least some long-term (e.g., years) effect of adversity during childhood on HPA axis function in childhood and adolescence.

The final consideration is whether the cortisol profiles that are present in the data can be applied to the ACM and are representative of the ACM's unemotional, buffered, and vigilant pattern as alluded to previously in this paper. The cortisol profiles that emerged from the data were defined and labeled according to their relation to the other profiles in the data and not by referencing empirical research on what constitutes a blunted, moderate, or elevated response because that information is not available. Therefore, it is not clear whether the three cortisol profiles map directly onto the ACM patterns or if the patterns would have the same meaning in relation to the entire child and adolescent population's cortisol reactivity pattern. This hypothesis is not possible to test with the current data but may eventually be tested with a larger and more diverse sample that includes children and adolescents with a wider variety of adverse experiences.

There are also considerable strengths to the study in spite of limitations. To date, this is the first study that longitudinally tested for the four hypothesized SRS patterns as described by the ACM (Del Giudice et al., 2011) in maltreated and nonmaltreated youth across the important developmental period of adolescence. While according to the ACM, a downregulated SRS is considered adaptive for individuals habitually exposed to stress, over time decreased levels of glucocorticoids are thought to contribute to disease states, including autoimmune disease such as arthritis (Tsigos \& Chrousos, 2002), highlighting the clinical relevance of the findings. The known interactions between the stress and immune systems suggest that immune system markers such as cytokines should be considered along with stress system bio-markers.

The findings from this study provide a foundation from which to design and implement future studies to test for adaptive calibration. Future studies should work toward pinpointing the time scale on which adaptive calibration takes place and determine whether there are sensitive periods of development during which intervention has the greatest impact on SRS function in order to prevent future health and behavior problems. For example, current work on resiliency in animal models suggests there is a loss of reversibility with aging and that early life experiences influence plasticity of the SRS to future stressors (for review, see McEwen et al., 2015). By collecting measures of SRS function at more regular intervals (e.g., annually) over an extended period of time during known sensitive periods of HPA axis development (e.g., during the transition from childhood to adolescence; Lupien et al., 2009; Romeo \& McEwen, 2006), we may be able to better identify youth who would be good candidates for intervention by targeting individuals within a certain time frame following maltreatment experiences. 


\section{Acknowledgments}

Support for this research was provided by National Institute of Child Health and Human Development Grant R01 HD 039129 and National Institute on Drug Abuse Grant R01 Da24569-01A2. We thank the children and their families who participated in this research.

\section{References}

Barnett, D., Manly, JT., Cicchetti, D. Defining child maltreatment: The interface between policy and research. In: Cichetti, D., Toth, SL., editors. Advances in applied developmental psychology: Child abuse, child development and social policy. Norwood, NJ: Ablex; 1993. p. 7-73.

Cicchetti D, Rogosch FA, Gunnar MR, Toth SL. The differential impacts of early physical and sexual abuse and internalizing problems on daytime cortisol rhythm in school-aged children. Child Development. 2010; 81:252-269. [PubMed: 20331666]

Del Giudice MD, Ellis BJ, Shirtcliff EA. The adaptive calibration model of stress responsivity. Neuroscience \& Biobehavioral Reviews. 2011; 35:1562-1592. [PubMed: 21145350]

Horowitz, L. Unpublished doctoral dissertation. Catholic University; Washington, DC: 1998. The relationship of childhood sexual abuse to revictimization: Mediating variables and developmental processes.

Kirschbaum C, Pirke K, Hellhammer DH. The "Trier Social Stress Test"-A tool for investigating psychobiological stress responses in a laboratory setting. Neuropsychobiology. 1993; 28:76-81. [PubMed: 8255414]

Leeb, RT., Paulozzi, L., Melanson, C., Simon, T., Arias, I. Child maltreatment surveillance: Uniform definitions for public health and recommended data elements, version 1.0. Atlanta, GA: Centers for Disease Control and Prevention, National Center for Injury Prevention and Control; 2008.

Lupien SJ, McEwen BS, Gunnar MR, Heim C. Effects of stress throughout the lifespan on the brain, behavior and cognition. Nature Reviews Neuroscience. 2009; 10:434-445. [PubMed: 19401723]

Marceau K, Ram N, Houts R, Grimm K, Susman EJ. Individual differences in boys' and girls' timing and tempo of puberty: Modeling development with nonlinear growth models. Developmental Psychology. 2011; 47:1389-1409. [PubMed: 21639623]

Margolin G, Gordis EB. The effects of family and community violence on children. Annual Review of Psychology. 2000; 51:445-479.

McEwen BS. Stress, adaptation, and disease: Allostasis and allostatic load. Annals of the New York Academy of Sciences. 1998; 840:33-44. [PubMed: 9629234]

McEwen BS. Allostasis and allostatic load: Implications for neuropsychopharmacology. Neuropsychopharmacology. 2000a; 22:108-124. [PubMed: 10649824]

McEwen BS. The neurobiology of stress: From serendipity to clinical relevance. Brain Research. 2000b; 886:172-189. [PubMed: 11119695]

McEwen BS. Physiology and neurobiology of stress and adaptation: Central role of the brain. Physiological Reviews. 2007; 87:873-904. [PubMed: 17615391]

McEwen BS, Gray JD, Nasca C. Recognizing resilience: Learning from the effects of stress on the brain. Neurobiology of Stress. 2015; 1:1-11. [PubMed: 25506601]

Mennen FE, Kim K, Sang J, Trickett PK. Child neglect: Definition and identification of youth's experiences in official reports of maltreatment. Child Abuse and Neglect. 2010; 34:647-658. [PubMed: 20643482]

Muthén, LK., Muthén, BO. Mplus user's guide. 7. Los Angeles: Author; 1998-2011.

Muthén, LK., Muthén, BO. Mplus user's guide. 6. Los Angeles: Author; 2007.

Noll JG, Horowitz LA, Bonanno GA, Trickett PK, Putnam FW. Revictimization and self-harm in females who experienced childhood sexual abuse: Results from a prospective study. Journal of Interpersonal Violence. 2003; 18:1452-1471. [PubMed: 14678616]

Nottelmann ED, Susman EJ, Dorn LD, Inoff-Germain G, Loriaux DL, Cutler G Jr, et al. Developmental processes in early adolescence: Relations among chronologic age, pubertal stage, height, weight, and serum levels of gonadotropins, sex steroids, and adrenal androgens. Journal of Adolescent Health Care. 1987; 8:246-260. [PubMed: 3583875] 
Peckins MK, Dockray S, Eckenrode JL, Heaton J, Susman EJ. The longitudinal impact of exposure to violence on cortisol reactivity in adolescents. Journal of Adolescent Health. 2012; 51:366-372. [PubMed: 22999837]

Ritchers JE, Saltzman W. Childhood victimization and violent offending. Violence and Victims. 1990; 5:19-35. [PubMed: 2278946]

Romeo RD, McEwen BS. Stress and the adolescent brain. Annals of the New York Academy of Sciences. 2006; 1094:202-214. [PubMed: 17347352]

Slyper AH. The pubertal timing controversy in the USA, and a review of possible causative factors for the advance in timing of onset of puberty. Clinical Endocrinology. 2006; 65:1-8. [PubMed: 16817811]

Sterling, P., Eyer, J. Allostasis: A new paradigm to explain arousal pathology. In: Fisher, S., Reason, J., editors. Handbook of life stress, cognition, and health. New York: Wiley; 1988. p. 629-649.

Stone AA, Schwartz JE, Smyth J, Kirschbaum C, Cohen S, Hellhammer D, et al. Individual differences in the diurnal cycle of salivary free cortisol: A replication of flattened cycles for some individuals. Psychoneuroendocrinology. 2001; 26:295-306. [PubMed: 11166492]

Susman EJ. Psychobiology of persistent antisocial behavior: Stress, early vulnerabilities and the attenuation hypothesis. Neuroscience \& Bio-behavioral Reviews. 2006; 30:376-389.

Trickett PK, Gordis E, Peckins MK, Susman EJ. Stress reactivity in maltreated and comparison male and female young adolescents. Child Maltreatment. 2014; 19:27-37. [PubMed: 24482544]

Trickett PK, Noll JG, Susman EJ, Shenk CE, Putnam FW. Attenuation of cortisol across development for victims of sexual abuse. Development and Psychopathology. 2010; 22:165-175. [PubMed: 20102654]

Tsigos C, Chrousos GP. Hypothalamic-pituitary-adrenal axis, neuroendocrine factors and stress. Journal of Psychosomatic Research. 2002; 53:865-871. [PubMed: 12377295]

US Department of Health and Human Services, Administration for Children and Families, Administration on Children, Youth and Families, Children's Bureau. Child Maltreatment 2012. 2013. Retrieved from http://www.acf.hhs.gov/programs/cb/research-data-technology/statisticsresearch/child-maltreatment 


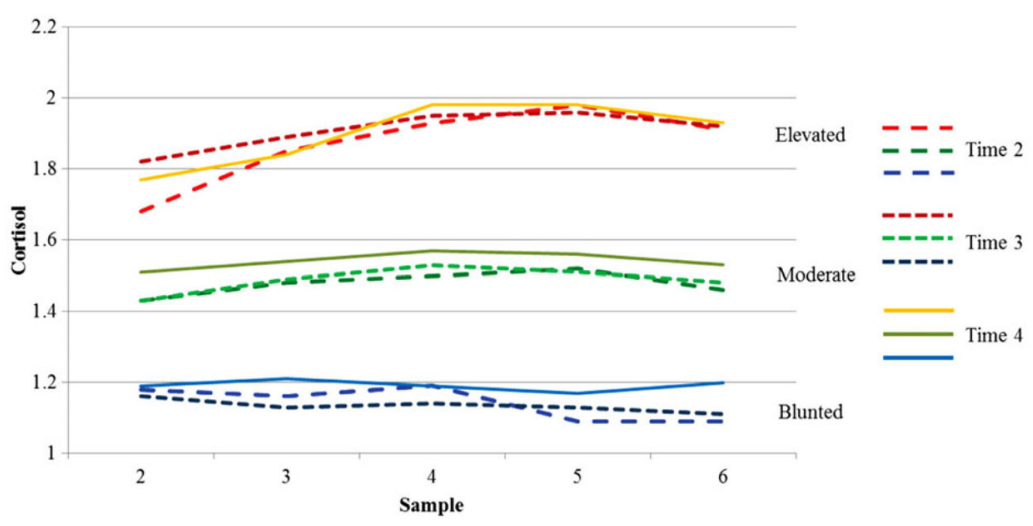

Figure 1.

Latent profile analysis three-profile solution at each time point. Cortisol, log transformed cortisol; blunted Time 2, $n=134$ (34\%); moderate Time 2, $n=214$ (55\%); elevated Time 2, $n=43$ (11\%); blunted Time 3, $n 125$ (39\%); moderate Time 3, $n=168$ (52\%); elevated Time 3, $n=29$ (9\%); blunted Time 4, $n=64$ (19\%); moderate Time 4, $n=179$ (52\%); elevated Time 4, $n=98$ (29\%). 


\section{Table 1}

Frequencies and percentages of categorical variables

\begin{tabular}{llrc}
\hline & Category & $\boldsymbol{n}$ & $\boldsymbol{\%}$ \\
\hline Child's sex & Male & 242 & 53.3 \\
& Female & 212 & 46.7 \\
Maltreatment status & Nonmaltreated & 151 & 33.3 \\
& Maltreated & 303 & 66.7 \\
Maltreatment type ${ }^{a}$ & Nonmaltreated & 151 & 33.3 \\
& Emotional abuse and/or neglect only & 118 & 26.8 \\
& Sexual and/or physical abuse & 181 & 39.9 \\
Child's race/ethnicity & Black & 171 & 37.7 \\
& White (non-Hispanic) & 50 & 11.0 \\
& Hispanic & 177 & 39.0 \\
& Mixed/biracial & 56 & 12.3 \\
Racial/ethnic minority & White (non-Hispanic) & 50 & 11.0 \\
& Minority (Black, Hispanic, mixed/biracial) & 404 & 89.0 \\
Time 2 time of day $b$ & Between 12 p.m. and 4 p.m. & 279 & 71.5 \\
& Between 4 p.m. and 8 p.m. & 111 & 28.5 \\
Time 3 time of day $b$ & Between 12 p.m. and 4 p.m. & 265 & 83.1 \\
& Between 4 p.m. and 8 p.m. & 54 & 16.9 \\
& Between 12 p.m. and 4 p.m. & 275 & 82.6 \\
& Between 4 p.m. and 8 p.m. & 58 & 17.4 \\
\hline \multirow{2}{*}{ Time of day $b$} & &
\end{tabular}

Maltreatment type missing for $n=4$.

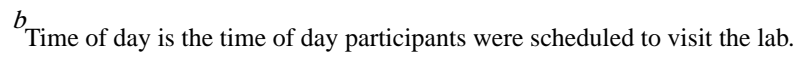


PECKINS et al. 
PECKINS et al.

Page 16

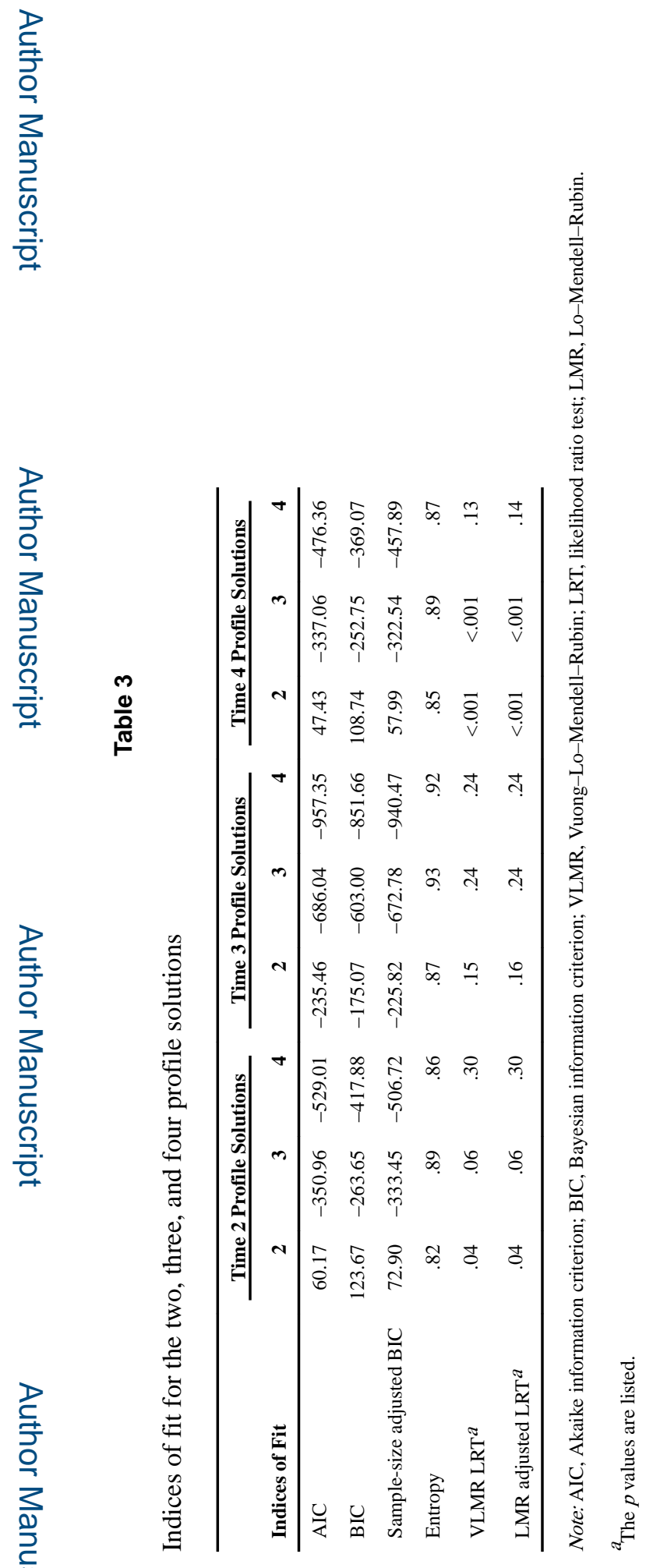

Lev Psychopathol. Author manuscript; available in PMC 2017 March 16. 


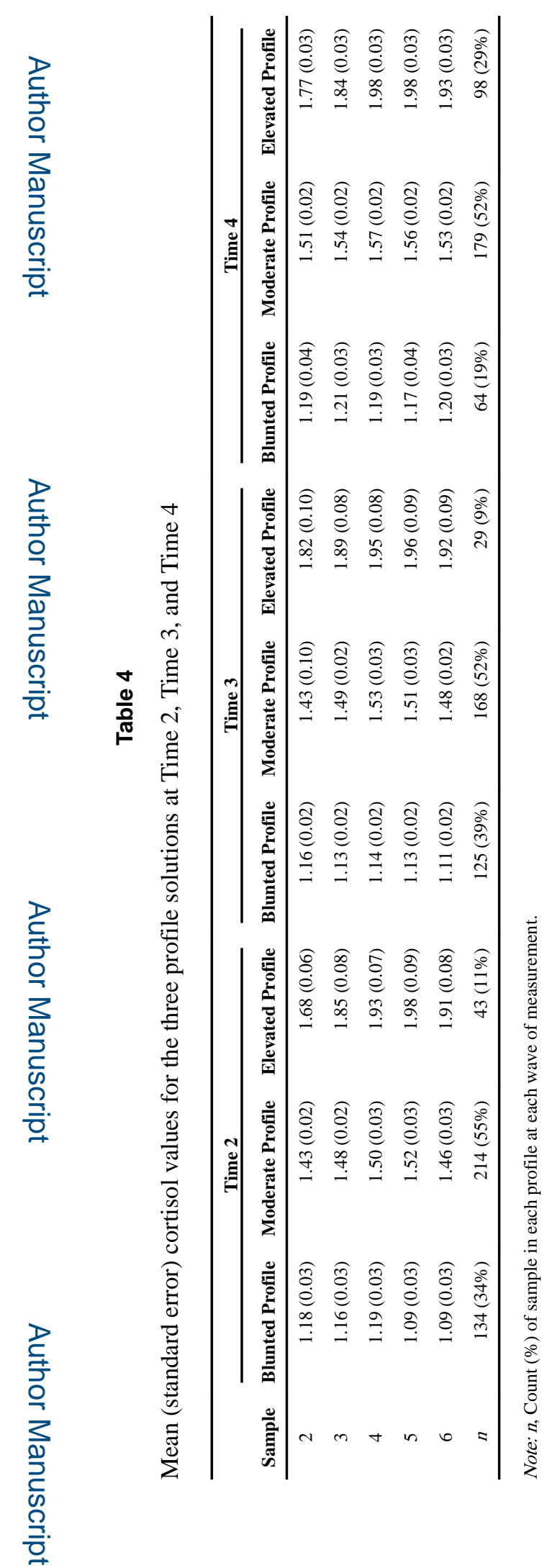


PECKINS et al.

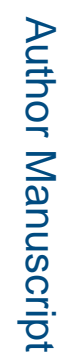

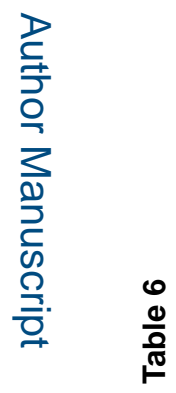

를

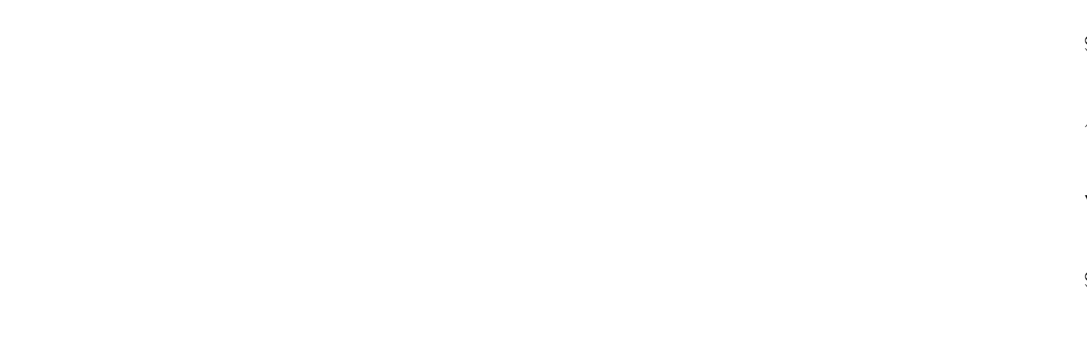

ֻٕ

落 